\title{
Formation of vortex clusters on a sphere
}

\author{
V. Pavlov ${ }^{1}$, D. Buisine ${ }^{1}$, and V. Goncharov ${ }^{2}$ \\ ${ }^{1}$ DMF, UFR de Mathématiques Pures et Appliquées, Université de Lille 1, 59655 Villeneuve d'Ascq Cedex, France \\ ${ }^{2}$ Institute of Atmospheric Physics, Russian Academy of Sciences, 109017 Moscow, Russia
}

Received: 4 October 1999 - Accepted: 25 April 2000

\begin{abstract}
This paper applies the Hamiltonian Approach (HA) to two-dimensional motions of incompressible fluid in curvilinear coordinates, in particular on a sphere. The HA has been used to formulate governing equations of motion and to interpret the evolution of a system consisting of $N$ localized two-dimensional vortices on a sphere. If the number of vortices $N$ is large, $N \sim 10^{2}-10^{3}$, a small number of vortex collective structures (clusters) is formed. The surprise is that a quasi-final state does not correspond to completely disorganized distribution of vorticity. Numerical analysis has been carried out for initial conditions taken in the form of $a$ few axisymmetric chains of point vortices distributed initially in fixed latitudes. The scheme of Runge-Kutta of 4 th order has been used for simulating an evolution of resulting flows. The numerical analysis shows that the Kelvin-Helmholtz instability appears immediately formating initial disorganized structures which are developed and finally "bursted". The system evolves to a few separated vortex "spots" which exist sufficiently for a long time.
\end{abstract}

\section{Introduction}

In the past, a number of theoretical and experimental studies have been devoted to the understanding of the dynamics of atmospheric and oceanic vortices which are frequently observed in nature (Fig.1). Vortices of planetary scale are interesting to a large extent because of their longevity, their robustness to perturbations, their coexistence with surrounding turbulence, and their ubiquity in laboratory, geo- and astrophysical flows. The interest in the problem is also driven by the anxiety of the civilization's impact on the environment (see, for example, McIntyre (1991) for a review on the ozone hole, or works on the stratospheric polar vortex).

Compared to the traditional fluid dynamics, atmospheric and oceanic vortex dynamics includes a number of physical restrictions (quasi-two-dimensional motion of wave and vortex structures on a curvilinear (spherical) surface, stratification, presence of shear flows, rotation of the Earth, etc.)

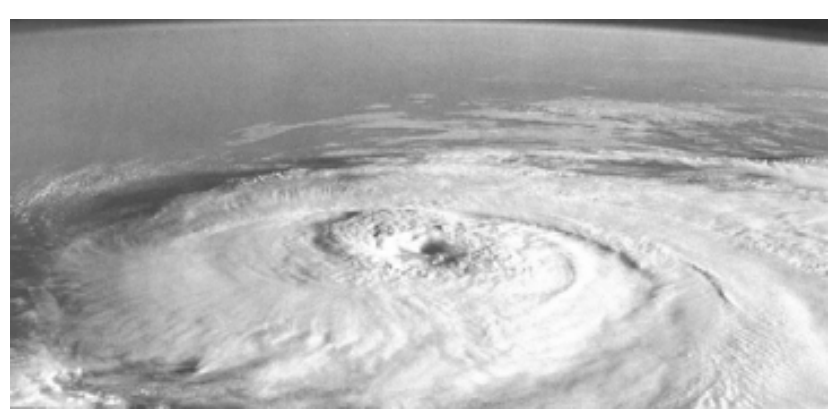

Fig. 1. Cyclone.

which strongly affect the motion and interaction of vortex fields and vortices. A study combining all these factors is a very complicated problem, and therefore until now has been frequently investigated separately, or with some simplified assumptions.

There have been numerous studies of the stability, interactions, and mergers of small numbers of isolated vortices (see Overman and Zabusky, 1982, and Refs therein). An evolution of large-scaled vortices have been discussed in the works of Bogomolov (1977), Zabusky and McWilliams (1982), Reznik (1992) and Polvani and Dritschel (1993) (see Refs therein also). Marcus (1990) has carried out dynamical simulation calculations (concerning the Great Red Spot which can be considered as a persistent spot of cyclonic vorticity) on a flat annulus with rigid boundaries. The effect of planetary curvature and rotation was incorporated by the $\beta$-plane approximation. In the model of Miller et al. (1992), the Monte Carlo simulations were compared with the long-time dynamics of Marcus (1990). Smith and O'Neill (1990) confined their research to the point-vortex limit (see Miller et al., 1990). The list of similar works can be continued.

With few exceptions, most of the works on this topic are focused generally on the development of complex three-dimensional numerical models. However, these giant models have somehow obscured the fact that a number of fundamen- 
tal responses can be found from simpler models that possess distinct physical fundamentals. It should be kept in mind that without addressing, in the beginning, the "big picture", the brute-force computer calculations of vortex phenomena for complex configurations are not very appealing. Their results often depend on a number of factors of secondary importance which can both distort the overall picture and introduce details frequently even nonexistent.

With this in mind, we extend in the present work the study of the Hamiltonian dynamics of vortices ${ }^{1}$ in a two-dimensional thin layer of incompressible fluid by examining their behavior when they are moving on the curved surface.

Thus, in the following consideration, there are two conceptual aspects: a two-dimensional approximation of hydrodynamical motions, and the use of the Hamiltonian Approach.

Let us briefly review some of the key factors of the twodimensional hydrodynamic models (for detailed discussion and justification of the models, the reader can be directed to classical textbooks, for example, Pedlosky (1987)).

Let the fluid motions be characterized by the following typical scales: the horizontal, $V$, and vertical, $W$, velocities of the fluid, as well as the horizontal length-scale, $L$, of a vortex structure. The layer thickness, $D$, will be regarded as the vertical length-scale. If the Froude number, $F r=V^{2} / g L$, is small, and the Reynolds number, $R e_{D}=V D^{2} / \nu L$ is large, the problem can be considered as two-dimensional and inviscid. Here $g$ is the gravity acceleration, and $v$ is the kinematic viscosity of the fluid. Here the dominant mechanism of bottom Rayleigh friction is taken into account (Pedlosky, 1987, and Refs therein). If the horizontal size of the vortex structure has a scale of $L \sim 10^{4} \div 10^{6} \mathrm{~m}$, the thickness of the atmosphere is of $D \sim 10^{4} \mathrm{~m}$, then for a moderate velocity of flows $V \sim 1 \div 10^{1} \mathrm{~ms}^{-1}$ and typical viscosity $v \sim 10^{-5} \mathrm{~m}^{2} \mathrm{~s}^{-1}$, the characteristic Reynolds number is $R e_{D}>10^{6} \gg 1$. Thus, the inequalities $F r \ll 1, R e_{D} \gg$ $1, D \ll L, W \ll V$ hold true, and we can consider the fluid layer as "thin", and motions as two - dimensional. Such estimations of dimensionless numbers are typical for geophysical hydrodynamics (Holton, 1992). Obviously, in venturing applications to the Earth's conditions, the neglected effects of dissipation, radiation and vertical structure distance this idealized model from the real thing. However, certain important features of the complex dynamics of vortex motion can be captured by this simple model.

The vorticity in real atmospheric and oceanic eddies often largely exceeds the background vorticity (for example, in oceanic rings or typhoons). Such localized vortex structures have a relatively large life-time. On the other hand, in the approximation of $R e^{-1} \rightarrow 0$ when a fluid is considered as inviscid, the governing hydrodynamical equations admit the singular vortices as its solution. Thus, point (singular) vortices, as a mathematical model, can be used as basic elements in a constructed model.

\footnotetext{
${ }^{1}$ There exists a few versions of the Hamiltonian Approach. We use the version formulated in the works of Goncharov and Pavlov (1993).
}

In spite of some difficulties in the interpretation of physical results, the concept of localized (in particular, singular) vortices is largely used in problems of geo- and astrophysical hydrodynamics. Singular vortices offer a simplified description, valid when the vortices are concentrated and well separated. In recent years a large number of publications have appeared in which different models of behavior of localized vortices have been proposed. For various reasons such models were found attractive. In many cases the study of the dynamics of the localized vortices and their interaction is simpler than in analogous problems for continuous vortex distribution. An arbitrary initial hydrodynamical field can be represented in the form of superposition of fields generated by such vortices, etc.

In the framework of the presented approximation, the vortex field evolution can be interpreted as a result of the interaction of the localized vortices, and the averaged vorticity is defined via their superposition.

In our work, we assume that the physics of the considered processes is described by inviscid Euler equations in two dimensions. In this case, 2D Euler flows can be described by a system of point vortices: the full vorticity is given by

$\Omega=\sum_{i} \gamma_{i} \delta^{(2)}\left(\boldsymbol{x}-\boldsymbol{x}_{i}\right)$.

Obviously, a model of point vortices provides a convenient mathematical model where one neglects the effects of finite vortex cores. (Effects of finite-area vortices are frequently addressed using uniform vortex patches, but neither system - point-vortices or vortex patches - is entirely satisfactory). In astrophysics, a similar double standard is used. For example, point masses are considered to describe problems of celestial mechanics, but fluid globes are used to model stellar structure.

Let us make here some comments about the difficulties associated with the concept of singular vortices.

(i) In the traditional description of experimental results, flows involve continuous distributions of vorticity. How can one approximate a continuous distribution of vorticity by means of point vortices? The response can be as follows: the physical meaning has only the averaged distribution $\langle\Omega\rangle=\int_{\Sigma} d \sigma \Omega$ where the characteristic size of the domain of space averaging, $(\Sigma)^{1 / 2}$, satisfies the condition $(D / N)^{1 / 2} \ll(\Sigma)^{1 / 2} \ll D^{1 / 2}$. Here $N$ is the full number of vortices, and $D$ the area of the domain where the vortices are localized. The averaging procedure has to be applied after calculating $\Omega$ only.

(ii) For a vorticity field consisting of point vortices, integrals of any finite power of the vorticity involve powers of delta-functions and are therefore singular. Yet in typical physical models the vorticity field has perfectly, well-defined moments. How can one define these integrals and moments?

The interpretation of the integrals is simple if we use the following consideration (see Landau and Lifshitz, 1987):

$$
\int d x \delta^{n}(x)=\lim _{\epsilon \rightarrow 0} \int d x \delta_{\epsilon}^{n-1}(0) \delta(x)
$$


Here, $\delta_{\epsilon}(x)$ is a "spreaded" delta-function with $\delta_{\epsilon}(0)=\epsilon^{-1}$ and the condition of normalization $\int d x \delta_{\epsilon}(x)=1 . \epsilon$ is the characteristic scale of the domain where the "spreaded" deltafunction is localized. Thus, we obtain

$\int d x \delta^{n}(x)=\epsilon^{-(n-1)} \int d x \delta(x)=\epsilon^{-(n-1)}$.

If $\Omega(\boldsymbol{x})=\sum_{i=1}^{N} \gamma_{i} \delta^{(2)}\left(\boldsymbol{x}-\boldsymbol{x}_{i}\right)$ (for the two-dimensional description), we obtain the estimate for the moment

$M^{(n)}=\int d \boldsymbol{x} \Omega^{n}(\boldsymbol{x})=\sum_{i=1}^{N} \gamma_{i}^{n} \sigma^{-(n-1)}$.

Here, $\sigma$ is the characteristic area of the domain occupied by a localized vortex. The number $N$ of vortices is fixed by the number of moments $M^{(n)}$ in question. Parameters $\gamma_{i}$ are found by solving the set of equations

$\sum_{i=1}^{N} \gamma_{i}^{j}=\sigma^{j-1} M^{(j)}, \quad j=1,2, \ldots N$.

In Section 2 we present the fundamentals of the Hamiltonian Approach. The governing equations for vortices moving on a spherical surface are formulated in Section 3. Vortex interactions are examined in Section 4. We show how the vorticity rearranges itself forming large vortex clouds. Numerical calculations, for several initial distributions, show that such collective structures form taking the form of clusters, like galaxies emerging from a system of point masses. It is still a surprise that this regime does not correspond to a completely disorganized (homogeneous) distribution of vorticity. Our conclusions regarding the results and the use of the method are presented in Section 5.

\section{Basic equations}

\subsection{Hamiltonian approach}

The second important aspect of the present work consists in the application of the Hamiltonian Approach (the version developed in works of Goncharov and Pavlov, 1993, 1997a,b) to the problems of the geophysical hydrodynamics. One of the motivations of the present work is connected with practical applications of this method.

Let us consider the dynamics of incompressible flows governed by equations

$\partial_{t} v_{\alpha}+v_{\beta} \partial_{\beta} v_{\alpha}=\partial_{\alpha}\left(\rho^{-1} p+\chi\right), \quad \partial_{\beta} v_{\beta}=0$,

where $v_{\alpha}$ are velocity components $(\alpha, \beta=1,2,3)$ in the Cartesian system of coordinates, $\partial_{t}$ is the partial derivative of a field variable with respect to time, $p$ is pressure, $\rho$ is density (further $\rho=1$ ). The summation convention is implied on repeated Greek indices when tensor notation is used.

The so-called hydrodynamical Hamiltonian systems evolve according to the law

$\partial_{t} u_{i}=\left\{u_{i}, \mathcal{H}\right\}=\int d \boldsymbol{x}^{\prime}\left\{u_{i}, u_{j}^{\prime}\right\} \frac{\delta \mathcal{H}}{\delta u_{j}\left(\boldsymbol{x}^{\prime}\right)}$, where the Hamiltonian, $\mathcal{H}$, of the system is the quantity functionally dependent on the fields, $u_{i}$. The Hamiltonian structure of hydrodynamical models consists, this way, of the Hamiltonian, $\mathcal{H}$, given by the total energy expressed in terms of field variables, $u_{i}$, and of the functional Poisson bracket $\{$,$\} .$ Conservation of energy follows from the given formulation, since $\partial_{t} \mathcal{H}=\{\mathcal{H}, \mathcal{H}\}=0$.

The detailed consideration of the subject has been given in the works of Goncharov and Pavlov (1993), Goncharov and Pavlov (1997a).

It is well known (Arnold, 1969, see also Morrison, 1982, Goncharov and Pavlov, 1993, 1997) that the system (1) may be presented on the phase space of the vorticity field

$\Omega_{\alpha}=\varepsilon^{\alpha \mu \beta} \partial_{\mu} v_{\beta}$

( $\varepsilon^{\alpha \mu \beta}$ is the Levi-Civita tensor) in Hamiltonian form as

$\partial_{t} \Omega_{\alpha}=\left\{\Omega_{\alpha}, H\right\}=\int d \boldsymbol{x}^{\prime}\left\{\Omega_{\alpha}, \Omega_{\beta}^{\prime}\right\} \frac{\delta H}{\delta \Omega_{\beta}^{\prime}}$.

Here and further, prime denotes that the field variables depend on space coordinate $\boldsymbol{x}^{\prime}$. The functional $H=H[\boldsymbol{\Omega}]$ under the symbol of the variational derivative $\delta / \delta \Omega_{j}$, the kinetic energy of flow:

$H=\frac{1}{2} \int d x v^{2}$

is the Hamiltonian. The skew-symmetric functional Poisson bracket in Eq. (4), $\left\{\Omega(\boldsymbol{x}), \Omega\left(\boldsymbol{x}^{\prime}\right)\right\}$, is local and is defined for the given model by expression

$\left\{\Omega_{\alpha}, \Omega_{\beta}^{\prime}\right\}=\varepsilon^{\alpha \sigma \gamma} \varepsilon^{\gamma \lambda \nu} \varepsilon^{\beta \nu \mu} \partial_{\sigma} \Omega_{l} \partial_{\mu} \delta\left(\boldsymbol{x}-\boldsymbol{x}^{\prime}\right)$.

Here and further, $d \boldsymbol{x}=d x_{1} d x_{2} d x_{3}$.

The curvilinear generalization of (6) and (5) in the case of when the used coordinates $\boldsymbol{x}=\left(x_{1}, x_{2}, x_{3}\right)$ are not Cartesian, may be written (see Goncharov and Pavlov, 1993) as

$$
\begin{aligned}
& \left\{\Omega^{\alpha}, \Omega^{\prime \beta}\right\}= \\
& \quad=g^{-1 / 2} \varepsilon^{\alpha \sigma \gamma} \varepsilon_{\gamma \lambda \nu} \varepsilon^{\beta \nu \mu} \partial_{\sigma} \Omega_{\lambda} \partial_{\mu} g^{-1 / 2} \delta\left(\boldsymbol{x}-\boldsymbol{x}^{\prime}\right), \\
& H=\frac{1}{2} \int d \boldsymbol{x} g^{1 / 2} g^{\alpha \beta} v_{\alpha} v_{\beta},
\end{aligned}
$$

where $g^{\alpha \beta}$ is the metric tensor, $g$ is its determinant, $\Omega^{\alpha}$ are contravariant components of the vorticity, $v_{\alpha}$ are covariant components of the velocity. Here,

$\Omega^{\alpha}=g^{-1 / 2} \varepsilon^{\alpha \beta \kappa} \partial_{\beta} v_{\kappa}$.

is the curvilinear generalization of (3).

\subsection{Two-dimensional approximation}

We will consider effectively two-dimensional incompressible flows whose particles move along non-intersecting stationary fluid surfaces. Depending on the symmetry of the problem, it is convenient to study such flows in a suitable system of orthogonal curvilinear coordinates $x_{1}, x_{2}, x_{3}$ so that coordinate lines $x_{3}$ coincide with vortex ones while coordinate lines 
$x_{1}$ and $x_{2}$ lay on the fluid surfaces. In such coordinate systems the vector field of the velocity has two components $\boldsymbol{v}=$ $\left\{v_{1}, v_{2}, 0\right\}$ and the vector field of the vorticity has only one component $\boldsymbol{\Omega}=\{0,0, \Omega\}$, where $\Omega=g^{-1 / 2}\left(\partial_{1} v_{2}-\partial_{2} v_{1}\right)$.

The incompressibility equation, $\boldsymbol{\nabla} \cdot \boldsymbol{v}=0$, which is presented obviously in the form

$g^{-1 / 2} \partial_{\beta}\left(g^{1 / 2} v^{\beta}\right)=0$.

allows us to introduce the stream function $\Psi$

$v^{\beta}=g^{-1 / 2} \varepsilon^{\beta \alpha} \partial_{\alpha} \Psi$,

where $\varepsilon^{\alpha \beta}$ is an anti-symmetrical unit tensor of second order, $\varepsilon^{12}=1, \varepsilon^{21}=-1$.

Let us note here that $\Psi$ possess symmetry $\Psi \rightarrow \Psi+C^{t e}$, i.e. the coordinate-independent part of the stream function $\langle\Psi\rangle$ may be omitted.

By virtue of the incompressibility condition the basic quantities can be expressed in terms of the stream function $\Psi$ as

$v_{1}=g_{11} g^{-1 / 2} \partial_{2} \Psi, \quad v_{2}=-g_{22} g^{-1 / 2} \partial_{1} \Psi ;$

$\Omega=-g^{-1 / 2}\left(\partial_{1} g_{22} g^{-1 / 2} \partial_{1}+\partial_{2} g_{11} g^{-1 / 2} \partial_{2}\right) \Psi$.

In the two-dimensional case the Poisson bracket (8) reduces to the more simple expression:

$\left\{\Omega, \Omega^{\prime}\right\}=\frac{\varepsilon^{\alpha \beta}}{\sqrt{g}} \frac{\partial \Omega}{\partial x_{\alpha}} \frac{\partial}{\partial x_{\beta}} \frac{\delta\left(\boldsymbol{x}-\boldsymbol{x}^{\prime}\right)}{\sqrt{g}}, \quad(\alpha, \beta=1,2)$.

To avoid confusion, it should be noted that contravariant vorticity $\Omega$ is named later merely as a vorticity and differs from the usual physical vorticity $\Omega g^{1 / 2}\left(g_{11} g_{22}\right)^{-1 / 2}$. Both definitions coincide only in cases when $g=g_{11} g_{22}$. The fundamental nature of contravariant vorticity is based on the fact that in two-dimensional incompressible non-plane flows only this characteristic is a scalar quantity which obeys to the law

$\partial_{t} \Omega+v^{\alpha} \partial_{\alpha} \Omega=0, \quad(\alpha=1,2)$.

That is, $\Omega$ is conserved conveying fluid particles along their Lagrangian trajectories.

Without the loss of generality we assume that $x_{1}$ coincides with streamlines of the unperturbed stationary problem. For this wide class of layer models, geometric properties of the space associated with such coordinate systems are characterized only by components $g_{11}, g_{22}, g_{33}$ of the metric tensor and by its determinant $g$ which are deemed independent of $x_{1}$, just as the velocity profile of the unperturbed flow.

Using equation (11), the Hamiltonian $\mathcal{H}^{*}$ may be rearranged as

$$
\begin{aligned}
\mathcal{H}^{*} & =\frac{\rho}{2} \int d \zeta(\nabla \Psi)^{2}=-\frac{\rho}{2} \int d \zeta \Psi \Omega \\
& =-\frac{\rho}{2} \int d \zeta^{1} d \zeta^{2} g^{1 / 2} \Psi \Omega,
\end{aligned}
$$

where

$\Omega=-g^{-1 / 2} \varepsilon^{\beta \alpha} \partial_{\alpha} u_{\beta}=-\Delta \Psi$.
Here, quantity $\Omega$ is a generalized vortex on a non-flat, twodimensional flow, $u_{\beta}$ are covariant components of the hydrodynamical velocity, $u_{\beta}=g_{\beta \alpha} u^{\alpha}, \Delta$ is a two-dimensional operator, similar to the Laplace operator, defined by:

$$
\begin{aligned}
\Delta & =g^{-1 / 2} \varepsilon^{\alpha \beta} \partial_{\beta} g_{\alpha \gamma} g^{-1 / 2} \varepsilon^{\gamma \nu} \partial_{v} \\
& =g^{-1 / 2}\left(\partial_{1} g_{22} g^{-1 / 2} \partial_{1}+\partial_{2} g_{11} g^{-1 / 2} \partial_{2}\right)
\end{aligned}
$$

In expression (15), the space-independent part of $\Omega$, i.e. $\langle\Omega\rangle$, can be eliminated too, because of the condition on the stream-function: $\int d \zeta \Psi\langle\Omega\rangle=\langle\Omega\rangle \int d \zeta \Psi=0$.

The coordinate-dependent part of stream function $\Psi$ can be expressed in terms of the full vortex field, $\Omega$. From equation (16) we find the relationship between these characteristics of field, i.e. between $\Psi$ and $\Omega$ :

$\Psi=-\int d \zeta^{\prime} G\left(\zeta, \zeta^{\prime}\right) \Omega\left(\zeta^{\prime}\right)$,

Here, Green's function $\mathcal{G}\left(\zeta, \zeta^{\prime}\right)$ is a solution of the equation

$\Delta G\left(\zeta, \zeta^{\prime}\right)=\delta^{(2)}\left(\zeta, \zeta^{\prime}\right)-V^{-1}$,

where $V$ is the "volume" of a domain where the deltafunction is defined (for a spherical surface, the "volume" is $V=4 \pi R^{2}$ and the concrete structure of $\mathcal{G}$ is given below). This result follows from the fact that the surface of a sphere, for example, has a "volume", but has no boundaries. If $V \rightarrow \infty$, one has the traditional equation. ${ }^{2}$ Applying operator $\Delta$ to expression (19), we find the correct relationship, $\Delta \Psi=\Omega-\langle\Omega\rangle$.

The kinetic energy of fluid may be written in the form

$\mathcal{H}^{*}=-\frac{\rho}{2} \iint d \zeta d \zeta^{\prime} \Omega \Omega^{\prime} \mathcal{G}\left(\zeta, \zeta^{\prime}\right)$.

If in the considered system there exist localized zones of intensive concentration of vorticity, the full stream function (vorticity field) may be presented as a sum of a regular part of the field, $\Psi^{r}$ (or $\Omega^{r}$ ), and a singular one, $\Psi^{s}\left(\Omega^{s}\right)$, associated with localized vortices (see the following section).

The Hamiltonian, $\mathcal{H}^{*}$, (integral of motion, energy expressed in terms of the canonical variables) is in this case

$$
\begin{aligned}
\mathcal{H}^{*}= & \frac{\rho}{2} \int d \zeta\left(\nabla \Psi^{r}\right)^{2}-\rho \sum_{i-1}^{N} \gamma_{i} \Psi^{r}\left(\zeta_{i}\right) \\
& -\frac{\rho}{2} \sum_{i, j} \gamma_{i} \gamma_{j} \cdot \mathcal{G}_{i j}
\end{aligned}
$$

The first term here represents the regular current energy, the second, $\mathcal{H}_{r s}$, describes the Hamiltonian of interaction between the regular current and localized vortices, and the last

\footnotetext{
${ }^{2}$ For a two-dimensional ideal incompressible fluid, we have $\Delta \Psi=\Omega$, for quasi-geostrophic flows in the barotropic atmospheric model, taking into consideration its "compressibility" and the so-called gyroscopic rigidity, and for the ocean $\left[\Delta-R^{-2}\right] \Psi=$ $\Omega$. Here, $\Omega$ is the potential vorticity, $R=\sqrt{g H} / \beta$ is the Obukhov scale (Obukhov, 1949), $H$ is the ocean depth or the characteristic atmospheric height, $g$ is the gravitational acceleration, and $\beta$ is the Coriolis parameter. Operator $\Delta$, the Laplacian, is written in the spherical metric.
} 
term $\mathcal{H}_{\text {int }}$ describes the interaction between localized vortices.

Thus, the study of the motion of two-dimensional vortices when they are interacting among themselves is assured by the Hamiltonian $\mathcal{H}_{\text {int }}$. Their behavior when they interact and are embedded, for example, in regular shearing zonal flows is described by $\mathcal{H}_{r s}+\mathcal{H}_{\text {int }}$, etc.

\subsection{Point vortices}

Consider now, in the framework of such a modified Hamiltonian formulation, an evolution of a system consisting of $N$ singular vortices: two-dimensional models of perfect fluid permit the existence of singular (point) vortices.

We assume that the vortex system consists of a linear superposition of point "sources", and the field, $\Omega$, is characterized by the following distribution of vorticity

$$
\begin{aligned}
\Omega & =\sum_{i} \gamma_{i} \delta^{(2)}\left(\zeta-\zeta_{i}(t)\right) \\
& =g^{-1 / 2} \sum_{i} \gamma_{i} \delta^{(1)}\left(\zeta^{1}-\zeta_{i}^{1}(t)\right) \delta^{(1)}\left(\zeta^{2}-\zeta_{i}^{2}(t)\right)
\end{aligned}
$$

In this case, a total vorticity is given by

$$
\int d \zeta^{\prime} \Omega\left(\zeta^{\prime}\right)=\sum_{i} \gamma_{i}
$$

Here, $\gamma_{i}$ are independent of time intensities of the vortices, $\left[\gamma_{i}\right]=L^{2} T^{-1}, \quad \zeta_{i}=\left(\zeta_{i}^{1}, \zeta_{i}^{2}\right)$ are their coordinates dependent on time, $\delta^{(2)}\left(\zeta-\zeta_{i}\right)$ is the two-dimensional function of Dirac (see (A10)), $\zeta_{i}=\zeta_{i}(t)$.

Notwithstanding this, let us agree that in this chapter the repetitive index will not mean a summation which will be shown as $\sum$.

Calculating the corresponding Poisson brackets $\left\{\zeta_{i}^{\alpha}, \zeta_{j}^{\beta}\right\}$ which follows directly from (14) and (22) (see also the definition of the delta-function), we find

$\left\{\zeta_{i}^{\alpha}, \zeta_{j}^{\beta}\right\}=\gamma_{i}^{-1} g_{i}^{-1 / 2} \delta_{i j} \varepsilon^{\alpha \beta}$

where $g_{i}=g\left(\zeta=\zeta_{i}\right)$ is calculated in the point where the vortex is localized.

Thus, in terms of variables $\zeta_{i}^{\alpha}$ the dynamics of a system of singular vortices will be described by the equations

$\partial_{t} \zeta_{i}^{\alpha}=\left\{\zeta_{i}^{\alpha}, \mathcal{H}\right\}=\frac{\varepsilon^{\alpha \beta}}{\gamma_{i} g^{1 / 2}} \frac{\partial \mathcal{H}}{\partial \zeta_{i}^{\beta}}$,

where canonical pair of dynamical variables is $\zeta_{i}^{\alpha},\{.,$.$\} is$ the functional Poisson bracket expressed in terms of variational derivatives, $\delta / \delta \zeta_{i}^{\alpha}, \mathcal{H}$ is the Hamiltonian, i.e. the full energy of the fluid expressed in terms of canonical variables. The Hamiltonian does not depend explicitly on time, and thus it is an integral of motion: $\partial_{t} \mathcal{H}=\{\mathcal{H}, \mathcal{H}\}=0$.

Using Green's function $\mathcal{G}\left(\zeta, \zeta^{\prime}\right)$, which satisfies the equation

$\Delta \mathcal{G}\left(\zeta, \zeta^{\prime}\right)=\delta^{(2)}\left(\zeta, \zeta^{\prime}\right)-V^{-1}$, we can find for the Hamiltonian of localized vortices

$\mathcal{H}=-\frac{1}{2} \sum_{i, j}^{N} \gamma_{i} \gamma_{j} \mathcal{G}\left(\zeta_{i}, \zeta_{j}\right)$

The final expression for $\mathcal{H}$ via the implicit Green's function is obtained by substituting (22) into (20).

The expression for $\mathcal{H}$ has a shortcoming by having an uncertainty which arises from turning into infinity of the energy of the interaction when $i=j$. We can write

$\mathcal{H}=\mathcal{H}_{i}+\mathcal{H}_{\text {int }}=-\frac{1}{2} \sum_{i} \gamma_{i}^{2} G_{i i}-\frac{1}{2} \sum_{i \neq j}^{\prime} \gamma_{i} \gamma_{j} G_{i j}$

One can show that the first term, $\mathcal{H}_{i}$, has a logarithmic divergence. But this term can be excluded from the consideration, because it is independent on the space coordinates due to the independence of $G_{i i}$ on the coordinates. Spherical coordinates may be considered as an example of a system of curvilinear coordinates which satisfy this requirement. ${ }^{3}$

\section{Governing equations for vortices on the sphere}

We consider the dynamics of an incompressible, unforced, inviscid and thin fluid shell on the spherical surface of radius $R$. We will work through our article in a frame of reference that is fixed in space. This formulation is opposed to the more common geophysical conventions considering a frame rotating with the system (the Earth, for example).

We suppose that the reader should not be confused as to whether the vortex dynamics presented below is in a rotating fluid or not. It is clear that a simple change of the coordinate system cannot affect the dynamics of the processes.

The dynamics of point vortices moving on a curvilinear surface is described by equations (25). On a sphere the location is given by longitude $\theta$ and latitude $\phi$. Coordinates $\zeta_{i}^{\alpha}$ are $\left(\theta_{i}, \phi_{i}\right)$, where $\alpha=1,2, g^{-1 / 2}=r^{2} \sin \theta$, tensor $\varepsilon^{\alpha \beta}$ has components $\varepsilon^{12}=-\varepsilon^{21}=1, \quad \varepsilon^{11}=-\varepsilon^{22}=0$. Spherical coordinates $r, \theta, \phi$ are connected with Cartesian ones as $x=r \sin \theta \cos \phi, \quad y=r \sin \theta \sin \phi, \quad z=r \cos \theta$. We have, thus, $\zeta^{1}=\theta, \zeta^{2}=\varphi, \zeta^{3}=r, g_{11}=r^{2}, g_{22}=$ $r^{2} \sin ^{2} \theta, g_{33}=1$, and $g=r^{4} \sin ^{2} \theta>0$. For a spherical surface the basic system of dimensionless equations becomes

$$
\begin{aligned}
\partial_{t} \theta_{i} & =\left(\gamma_{i} \sin \theta_{i}\right)^{-1} \partial_{\phi_{i}} \mathcal{H}_{i n t}, \\
\partial_{t} \phi_{i} & =-\left(\gamma_{i} \sin \theta_{i}\right)^{-1} \partial_{\theta_{i}} \mathcal{H}_{i n t} .
\end{aligned}
$$

\footnotetext{
${ }^{3}$ Obviously, only in the case when the character of these infinities does not depend on the location of the vortices, the self-action corresponding to the infinite energy which does not affect the evolution of the vortices may be excluded from the Hamiltonian (27). The mathematical nature of these infinities is universal. It is defined by the fact that when $\left|\zeta-\zeta^{\prime}\right| \rightarrow 0$ Green's function has a logarithmic divergence. Because the above-described divergence appears only under the assumption of singular vortices, when the vorticity distribution is described by the delta-function, this problem does not occur for objects of finite sizes.
} 


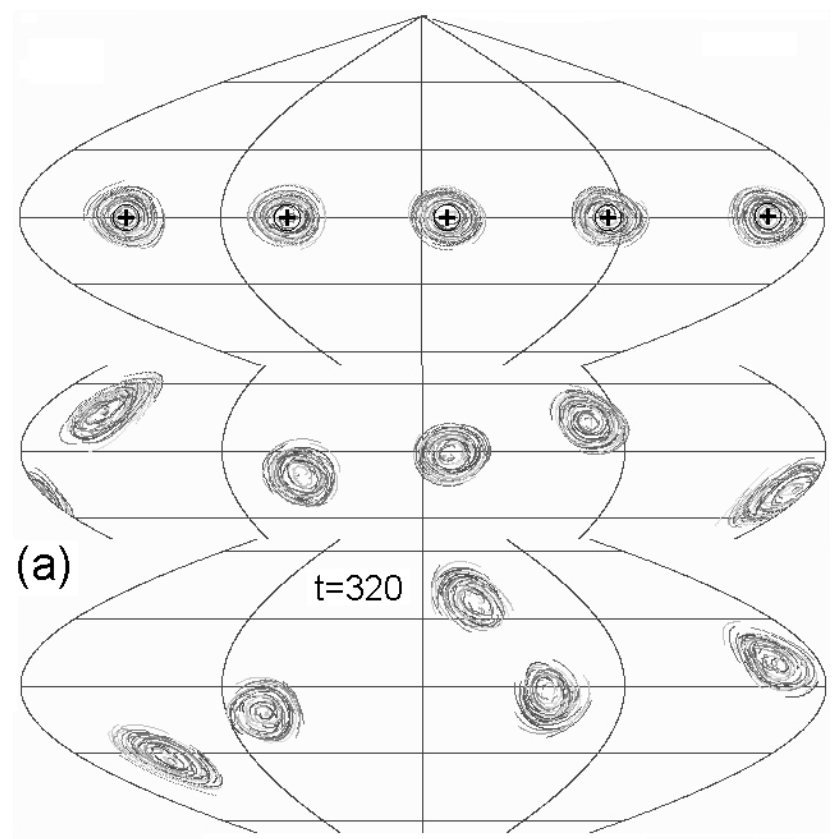

Fig. 2. The evolution of the system when $\mu=0.0613$. The distance between patches exceeds their initial diameter. The instability of the spot position is observed at the late stage of the simulation, approximately after 300 time steps.

Here, $\partial_{t}$ is the dimensionless derivative-operator with respect to time applied to a spherical coordinate of a vortex, time is measured in units of $\tau=r^{2} / \gamma_{0}, \gamma_{0}=\max \left(\left|\gamma_{i}\right|\right), \gamma_{i}=$ $\gamma_{i} / \gamma_{0},\left|\gamma_{i}\right| \leq 1, i=1, \ldots, N, \partial_{\phi_{i}}=\partial / \partial \phi_{i}, \partial_{\theta_{i}}=\partial / \partial \theta_{i}$, $0 \leq \theta \leq \pi, 0 \leq \phi-2 \pi k \leq 2 \pi, k=0, \pm 1, \pm 2, \ldots$.

If the regular current is absent, the Hamiltonian is given by the expression

$\mathcal{H}_{\text {int }}=-\frac{1}{2} \sum_{i, j} \gamma_{i} \gamma_{j} G_{i j}$

The diagonal terms are absent in the sum $\sum_{j, k}$.

The calculation of the Green's function (see Appendix B) give

$$
\begin{aligned}
G\left(\cos \beta_{i j}\right) & =-\frac{1}{4 \pi} \sum_{l=1}^{\infty} \frac{2 l+1}{l(l+1)} P_{l}\left(\cos \beta_{i j}\right) \\
& \equiv \frac{1}{4 \pi} \ln \left(1-\cos \beta_{i j}\right) .
\end{aligned}
$$

Here, $P_{l}(z)$ are Legendre polynomials. Obviously, the Green's function is defined up to a constant.

Let us note that if $z \rightarrow 1$, we obtain the approximation used frequently in plane motions. Evidently, in this case, $G(z) \simeq(4 \pi)^{-1} \ln \beta^{2}+\ldots$. This expression leads to the classical equations for vortices moving in the plane $x 0 y$ (evidently, in this case, one obtains $x_{k}=r \phi_{k}, y_{k}=$ $\left.r u_{k},\left|\phi_{k}\right| \ll 1,\left|u_{k}\right| \ll 1\right)$

$\gamma_{i} \partial_{t} y_{i}=-\partial_{x_{i}} \mathcal{H}_{i n t}, \quad \gamma_{i} \partial_{t} x_{i}=\partial_{y_{i}} \mathcal{H}_{\text {int }}$,

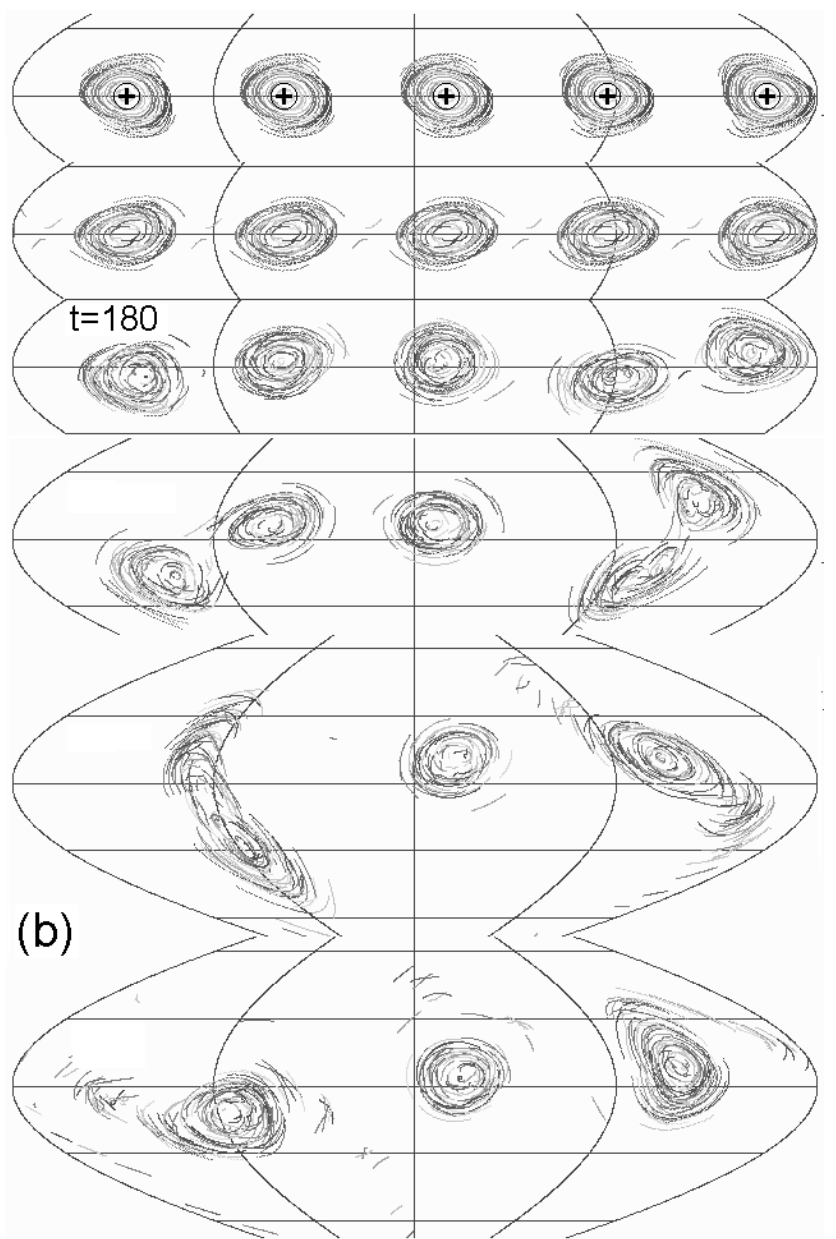

Fig. 3. The evolution of the system when $\mu=0.1$. The distance between patches is slightly less then their initial diameter. The instability is developed due to the intensive exchange of localized vortices.

where

$\mathcal{H}_{i n t}=-\frac{1}{4 \pi} \sum_{i \neq j}^{N} \gamma_{i} \gamma_{j} \ln \left[\left(x_{i}-x_{j}\right)^{2}+\left(y_{i}-y_{j}\right)^{2}\right]$,

and we arrive at the equations of motion of point vortices in the unbounded plane (see Lamb, 1932) ${ }^{4}$

\section{Results of calculations and discussion}

In our numerical calculations we use the expression for the Green's function given by (31). System of equations (29) is reduced to

\footnotetext{
${ }^{4}$ It is a classical result of Kirchhoff (1876) stating that the equations of a point-vortex motion can be written as $\gamma_{i} \partial_{t} x_{i}=$ $\partial \Psi / \partial y_{i}, \quad \gamma_{i} \partial_{t} y_{i}=-\partial \Psi / \partial x_{i}$, where the stream function $\Psi=$ $\Psi\left(x_{i}, y_{i}\right)$ is frequently called the "kinetic energy of interaction". In this connection, let us note that the detailed discussion on the problem of the motion of point vortices has been reviewed by Aref (1983).
} 


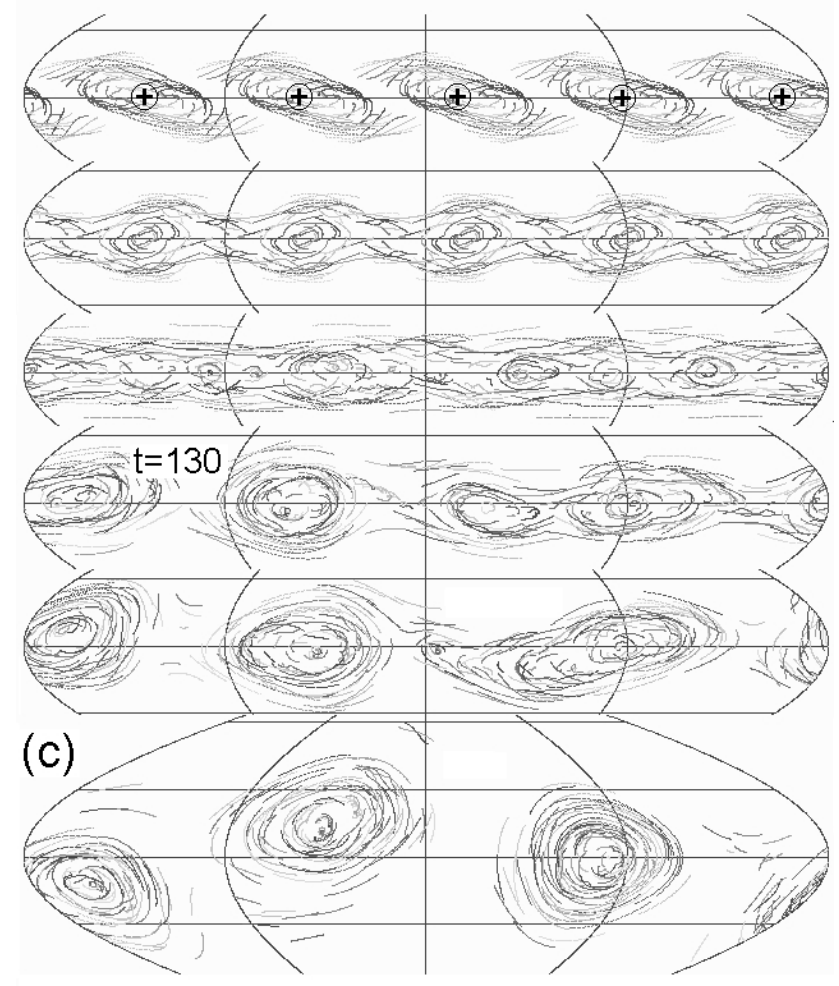

Fig. 4. The evolution of the system when $\mu=0.157$. The patches, which are initially at sufficiently short distances, exchange immediately with each other numerous vortices and merge forming rapidly a perturbed strip. Appearance of 2 or 4 structures is accompanied by the pushing out of vortices.

$$
\begin{aligned}
\partial_{t} \theta_{i}= & (4 \pi)^{-1} \sum_{j=1}^{N}{ }^{\prime} \gamma_{j}\left(1-\cos \beta_{i j}\right)^{-1} . \\
& \cdot \sin \theta_{j} \sin \left(\phi_{j}-\phi_{i}\right), \\
\partial_{t} \phi_{i}= & (4 \pi)^{-1} \sum_{j=1}^{N}{ }^{\prime} \gamma_{j}\left(1-\cos \beta_{i j}\right)^{-1}\left(\cos \theta_{j}\right. \\
& \left.-\sin \theta_{j} \cos \theta_{i} \sin ^{-1} \theta_{i} \cos \left(\phi_{j}-\phi_{i}\right)\right),
\end{aligned}
$$

where

$\cos \beta_{i j}=\sin \theta_{i} \sin \theta_{j} \cos \left(\phi_{j}-\phi_{i}\right)+\cos \phi_{i} \cos \phi_{j}$.

The system has been treated by means of a 4-order RungeKutta scheme. The time step is restricted by the condition $\Delta t<\eta \Delta \Psi / \sup \left(\left|\partial_{t} \theta_{i}\right|,\left|\partial_{t} \phi_{i}\right|\right)$ where $\Delta \psi$ is the characteristic angle between two point vortices. To avoid the polar singularity due to the metrics, a polar cap of 0,01 radian is excluded from the domain. The energy conservation has been evaluated during the calculations. The Hamiltonian $\mathcal{H}$ tries small variations: $-0.0192401 \leq \mathcal{H} \leq-0.0192344$, during the process of iterations from $t=0$ to $t=400$ with the the root-mean-square error $<3 \cdot 10^{-4}$.
The numerical analysis shows that the dynamical behavior of the structures in question includes an exchange of point vortices (dipoles) between clouds of vortices and the appearance of the fragmentation of the original structures, formed of initially regularly distributed vortices. We also analyze the fragmentation of an equatorial, initially homogeneous, jet.

We initialize the system of $m$ equidistant distribution of vortices centered at the latitude $\theta_{c}=\pi / 2$, and composed of $n^{2}$ point vortices. The parameters of the problem are: a) the fractional area $\mu$ occupied by the vortices: this parameter is defined as the total area enclosed by the vortex patches divided by $4 \pi: \mu \simeq m \delta \varphi \delta \theta \sin \theta_{c} / 4 \pi$ and b) the number $m$ of domains. The results of the numerical simulations are given in Figures $2-6$. The trajectories and the distribution density of the clouds of point vortices are given here in the framework of the so-called "sinus" - representation where the coordinates are defined by $x=\varphi \sin \theta$ and $y=\theta$.

The results are presented in Figures 2 - 4 for 5 equatorial patches each containing 64 point vortices and characterized by the parameters $\mu=0.0613,0.100$ and 0.157 .

Figure 2 shows the evolution of the system of vortices when $\mu=0.0613$. This parameter corresponds to the distance between patches exceeding their initial diameter. The instability of the spot position was observed at the late stage of the simulation, approximately after 300 time steps. This instability evidently is a result of the growing exponential of the accumulated numerical mistakes. The spots behave as isolated structures, and no exchange of vortices has been observed.

Figure 3 demonstrates the case of $\mu=0.1$. In this scenario the distance between the patches is slightly less than their initial diameter. In this case, from the very beginning, the episodical exchange of vortices is observed. After $t=180$ the instability of the positions appeared. This instability is developed due to the intensive exchange of localized vortices. 4 or 5 compact structures with densities similar to the ones in the initial configurations are formed. Finally, the system evolves into three patches. Several isolated point vortices ejected from the clusters are clearly observed. Figure 4 shows the evolution of the system when $\mu=0.157$. The patches, which are initially at sufficiently short distances, exchange immediately with each other numerous vortices and merge forming rapidly a perturbed strip which can be qualified as turbulent. Originating from this unstable strip, 4 structures are formed without "pushing out" of vortices at $t=130$. Later, the appearance of 2 or 4 structures is accompanied by the pushing out of vortices. Finally, 3 structures are formed with the vortex density comparable to one of initial clusters.

Let us present some results of the dynamic behavior of an equatorial jet consisting of $N=100$ point vortices. The intensities of the vortices are defined by the expression $\gamma_{i}=$ $\pm 1 / N \sin \theta_{i}$. These singular vortices are distributed along the strip with a transversal size $0.15 \pi$ radian, on circles of latitude $\theta_{i}=\pi \times\left(0.5+0.15 \xi_{i}\right)$, where $\xi_{i}=-0.5+(i-1) / 9$. This distribution corresponds to an equatorial jet with the following parameters: maximum of the velocity on the axis and 


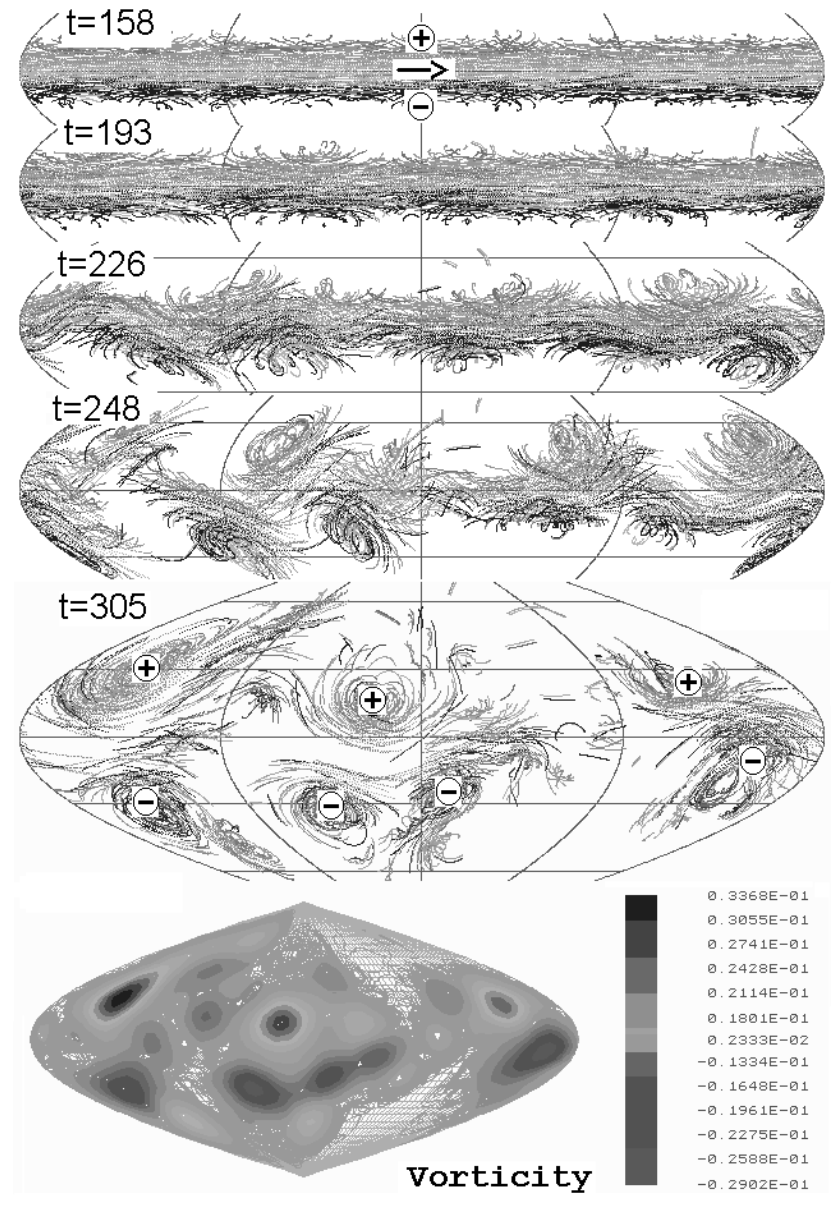

Fig. 5. Destruction of the strip and the formation of 3 or 4 clusters at $t=305$.

transversal variations of the velocity $\Delta V_{\phi, i}= \pm 0.1$. The sliding near the edges of the jet is the cause of the appearance of instability which is displayed for the first time at $t=158$ and $t=193$. Figure 5 illustrates fluctuations on the edges of the strip in a form of small quasi-symmetrical rings. These structures develop between $t=226$ and $t=246$. Very rapidly, appearing oscillations lead to the destruction of the strip and to the formation of 3 or 4 dipoles at $t=305$.

Finally, these structures develop into two large clusters of positive vorticity and three weak zones of negative vorticity $(t \geq 374)$ (Figure 6). These structures are accompanied by isolated vortices ejected during the process of transition.

The averaged space density of vortex distribution has been obtained from the following expression:

$\left\langle\omega\left(\boldsymbol{x}_{l}\right)\right\rangle=\sum_{i=1}^{N} \gamma_{i}\left\langle\delta\left(\boldsymbol{x}_{l}-\boldsymbol{x}_{i}\right)\right\rangle=\sum_{i=1}^{N} \gamma_{i} w\left(\beta_{l i}\right)$,

where $w\left(\beta_{l i}\right)=K \sup \left(1-\beta_{l i} / \pi v, 0\right), \beta_{l i} \equiv \beta\left[x_{l}, x_{i}\right]>$ $0, x_{l}$ is the coordinate of point $P_{l}, x_{i}$ is the coordinate of the $i^{\text {th }}$ singularity, $\beta\left[x_{l}, x_{i}\right]$ is the angle between the positions of $P_{l}$ and $P_{i}$. Constant $K$ is defined by the normalization condition: $K^{-1}=\int d \sigma w\left(\beta_{l i}\right)=\pi^{3} v^{2} / 3$. In our model calculations we used $v=10^{-1}$.

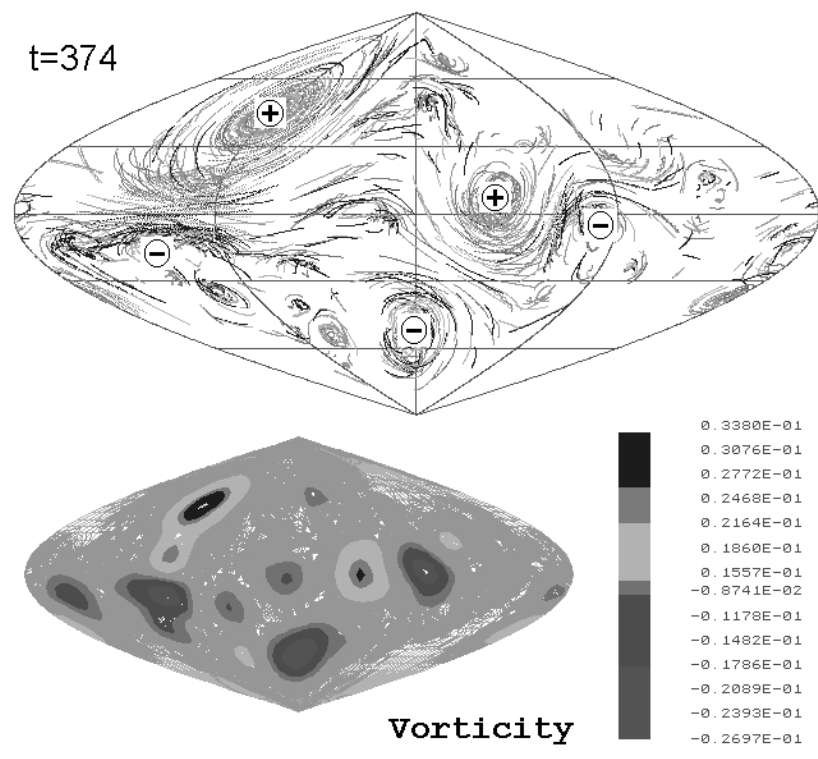

Fig. 6. Two large clusters of positive vorticity and three weak zones of negative vorticity are formed $(t \geq 374)$.

\section{Conclusion}

The paper was motivated by several reasons. First, the questions regarding what the Hamiltonian looks like and what the structure of the canonical equations is in concrete situations are not as trivial as they may appear at first glance. Indeed they must be addressed at the very beginning of the analysis of any practical application. Second, development of the Hamiltonian Approach would remain incomplete if no practical application of the theoretical analysis were given. As one of the important examples we consider a $2 D$ geophysical flow of an incompressible fluid.

As an application of the method, in this paper, we have examined two of the simplest configurations of flows on the surface of a sphere: a system of $N$ point vortices initially regularly distributed, and an equatorial jet. We have found that the vortex dynamics contains a change of vortices (vortex pairs) among vortex patches, with the appearance of fragmentation of the structures. Second, we have analyzed the stability and the fragmentation of the initially homogeneous equatorial jet. The most important result of the simulation is that the system forms cluster structures.

These obtained results are only indicative, since the proposed model is a very crude approximation of the real situation. However, it is interesting to note that in the framework of the proposed model we can explain the appearance of vortex structures. As the number of point vortices is increased, individual trajectories become of less concern because groups of many vortices are formed. With simple initial conditions these collective structures take the form of clusters, or regions of intense vorticity, like galaxies emerging from a system of point masses. The surprise is that this 
regime does not correspond to a completely disorganized (homogeneous) distribution of vorticity.

Let us conclude with a few remarks concerning the presented concept. In the current time of the intense use of computers, the merits of advanced analytical methods are often questioned: what good does a closed solution to a problem have, when it takes longer to analyze the problem analytically than to numerically integrate the underlying equations? In the response to such scepticism we can present the following argument.

Formal applications of finite-difference methods to systems of equations with Poisson brackets depending on fields (i.e. in non-canonical form) can lead to equations which will be not conservative. In such cases the loss of conservativity in Liuville's systems can be frequently observed. However, often such a loss of conservativity, as well as violation of the Jacobi property, may occur not due to the physical changes in the system, but rather due to the numerical errors accumulated in the finite-differential schemas (see comments in the work of Goncharov and Pavlov, 1997a). This remark is of particular significance because theoretical and computing physics widely uses discrete models with adequate correspondence to continuous analogies.

The analytical manipulations in the framework of the HA are not replicated according to the number of equations. The Jacobi's property which defines whether or not the system is Liuvillian is assured automatically. Indeed, in this approach, the object for approximating procedures is not the equations which are, as a rule, large in number, but a single quantity Hamiltonian, the full energy of the system presented in terms of canonical field variables.

Let us emphasize that the use of numerical methods imposes special requirements on the structure of the essential element of the method - the Poisson bracket (see, Goncharov and Pavlov, 1997a). It is evident that approximate methods are most effectively realized in the framework of the Hamiltonian (canonical) formulation with the Poisson tensor independent of field variables. In this case, there is only one object for approximation - the Hamiltonian, and corresponding calculations, which as a rule, could have a cumbersome, recurrent character, are not replicated in accordance to the number of equations.

The merits of the Hamiltonian method are the ease of transformation to new coordinates and the simplicity of perturbation calculus. The method is most convenient, not only for the derivation of the dynamic equations, but also for the estimate of the extent to which one or the other of the used approximations is universal. The method also allows one to reduce analytical manipulations to a minimum when solving concrete problems.

In conclusion, let us note that casting one's problem into the set of Hamilton's canonical equations, (here the conjugate variables are $p$ and $q) \partial_{t} p=-\delta H / \delta q, \quad \partial_{t} q=\delta H / \delta p$, brings benefits to the researcher. The computer is itself a "Hamiltonian device": it has a memory (denote its state by $F)$ and a central processor $(H)$ programmed to operate in each cycle $\delta t: \delta F=\delta t H(F)$. A simulation of the evo- lution of a system which follows Hamilton's equations is, therefore, an ideal task for a computer.

Acknowledgements. V. Goncharov express his gratitude to the UFR of Pure and Applied Mathematics and to LML (URA 1441) for its hospitality during his visit to the University of Lille 1 . This work was partially supported by the Russian Fundamental Research Foundation under grant No. 00-05-64019.

\section{Appendix A Spherical harmonics}

The appropriate orthogonal basis functions are the spherical harmonics $Y_{l m}(\zeta)$ which are defined here by the expression

$$
\begin{aligned}
& Y_{l m}(\theta, \phi)= \\
& \quad=(-1)^{m} i^{l}\left[\frac{(2 l+1)(l-m) !}{4 \pi(l+m) !}\right]^{1 / 2} P_{l}^{m}(\cos \theta) e^{i m \phi} .
\end{aligned}
$$

Functions $P_{l}^{m}(\cos \theta)$ are the associated Legendre functions of the first kind of degree $l$ (see below). It is required that $|m| \leq l$. For negative order $m$, the harmonics are defined by

$(-1)^{l-|m|} Y_{l,-|m|}(\theta, \phi)=Y_{l,|m|}^{*}(\theta, \phi)$,

Harmonics $Y_{l m}$ with $m<0$ are represented by (A1) where $|m|$ is used in place of $m$ and the coefficient $(-1)^{m}$ is omitted. It is clear that $m$ designates the zonal wave number, $l-|m|$ designates the number of nodes of $P_{l}^{m}$ in the interval $-1<$ $\cos \theta<1$ (i.e. between the poles) and, thus, measures the meridional scale of the spherical harmonics.

The structures of the first several spherical harmonics $Y_{l m}$ are given below (here $u=\cos \theta$ ):

$$
\begin{aligned}
& Y_{00}=\frac{1}{(4 \pi)^{1 / 2}}, \\
& Y_{10}=i\left(\frac{3}{4 \pi}\right)^{1 / 2} u, \\
& Y_{1, \pm 1}=\mp\left(\frac{3}{8 \pi}\right)^{1 / 2}\left(1-u^{2}\right)^{1 / 2} e^{ \pm i \phi}, \\
& Y_{20}=\left(\frac{5}{16 \pi}\right)^{1 / 2}\left(1-3 u^{2}\right), \\
& Y_{2, \pm 1}= \pm\left(\frac{15}{8 \pi}\right)^{1 / 2} u\left(1-u^{2}\right)^{1 / 2} e^{ \pm i \phi}, \\
& Y_{2, \pm 2}=-\left(\frac{15}{32 \pi}\right)^{1 / 2}\left(1-u^{2}\right) e^{ \pm i 2 \phi}, \\
& Y_{30}=-i\left(\frac{7}{16 \pi}\right)^{1 / 2} u\left(5 u^{2}-3\right), \\
& Y_{3, \pm 1}= \pm i\left(\frac{21}{64 \pi}\right)^{1 / 2}\left(1-u^{2}\right)^{1 / 2}\left(5 u^{2}-1\right) e^{ \pm i \phi}, \\
& Y_{3, \pm 2}=-i\left(\frac{105}{32 \pi}\right)^{1 / 2} u\left(1-u^{2}\right) e^{ \pm i 2 \phi}, \\
& Y_{3, \pm 3}= \pm i\left(\frac{35}{64 \pi}\right)^{1 / 2}\left(1-u^{2}\right)^{3 / 2} e^{ \pm i 3 \phi} .
\end{aligned}
$$


An important property of the spherical harmonics is that they satisfy the relationship

$\nabla^{2} Y_{l m}(\zeta)=-r^{-2} l(l+1) Y_{l m}(\zeta)$

where $\nabla^{2}$ is the full $3 D$ Laplacian.

The spherical harmonics are normalized by the condition

$\int d \zeta Y_{l m}(\theta, \phi) Y_{l^{\prime} m^{\prime}}^{*}(\theta, \phi)=r^{2} \delta_{l l^{\prime}} \delta_{m m^{\prime}}$,

with $d \zeta=r^{2} d \phi d \theta \sin \theta \equiv r^{2} d \Sigma$.

Thus, arbitrary field functions $\Psi(\theta, \phi)$ on the sphere are expanded in a series by letting

$\Psi(\theta, \phi)=\sum_{l=0}^{\infty} \sum_{m=-l}^{m=l} \Psi_{l m} Y_{l m}(\zeta)$,

$\Psi_{l m}=\int d \phi d \theta \sin \theta \Psi(\theta, \phi) Y_{l m}^{*}(\zeta)$.

From here, we can find

$$
\begin{aligned}
\Psi(\theta, \phi) \equiv & \int d \zeta^{\prime} \delta^{(2)}\left(\zeta-\zeta^{\prime}\right) \Psi\left(\theta^{\prime}, \phi^{\prime}\right) \\
= & \int d \phi^{\prime} d \theta^{\prime} \sin \theta^{\prime} r^{2} \Psi\left(\theta^{\prime}, \phi^{\prime}\right) \\
& \cdot(r)^{-2} \sum_{l=0}^{\infty} \sum_{m=-l}^{m=l} Y_{l m}(\zeta) Y_{l m}^{*}\left(\zeta^{\prime}\right)
\end{aligned}
$$

The spherical harmonic expansion of the delta-function is

$\delta^{(2)}\left(\zeta-\zeta^{\prime}\right)=r^{-2} \sum_{l=0}^{\infty} \sum_{m=-l}^{m} Y_{l m}(\zeta) Y_{l m}^{*}\left(\zeta^{\prime}\right)$.

The Dirac function $\delta^{(2)}\left(\zeta, \zeta^{\prime}\right)$ satisfies to the condition

$\int_{D_{1} \cap D_{2}} d \zeta^{\prime} \delta^{(2)}\left(\zeta, \zeta^{\prime}\right)=1$

This function is connected with the one-dimensional Dirac functions according to the relation

$\delta^{(2)}\left(\zeta, \zeta^{\prime}\right)=g^{-1 / 2} \delta^{(1)}\left(\zeta^{1}-\zeta^{1^{\prime}}\right) \delta^{(1)}\left(\zeta^{2}-\zeta^{2^{\prime}}\right)$,

where

$\int_{D_{1}} d \zeta^{1} \delta^{(1)}\left(\zeta^{1}-\zeta^{\prime \prime}\right)=\int_{D_{2}} d \zeta^{2} \delta^{(1)}\left(\zeta^{2}-\zeta^{2^{\prime}}\right)=1$

\section{Appendix B Green's function on a sphere}

For a spheric surface, we have $\zeta^{1}=\theta, \quad \zeta^{2}=\phi, \quad \zeta^{3}=$ $r, r=C^{t e}, g^{-1 / 2}=r^{2} \sin \theta>0$. Function $\mathcal{G}\left(\zeta, \zeta^{\prime}\right)$ satisfies the equation

$$
\begin{aligned}
& r^{-2}\left(\sin ^{-1} \theta \frac{\partial}{\partial \theta} \sin \theta \frac{\partial}{\partial \theta}+\sin ^{-2} \frac{\partial^{2}}{\partial \phi^{2}}\right) \mathcal{G}\left(\zeta, \zeta^{\prime}\right) \\
& =\delta^{(2)}\left(\zeta-\zeta^{\prime}\right)-\left(4 \pi r^{2}\right)^{-1}
\end{aligned}
$$

and can be expressed in terms of spherical harmonics $Y_{l m}(\zeta)$.
Function

$\mathcal{G}\left(\zeta, \zeta^{\prime}\right)=-\sum_{l=1}^{\infty} \sum_{m=-l}^{m} \frac{1}{l(l+1)} Y_{l m}(\zeta) Y_{l m}^{*}\left(\zeta^{\prime}\right)$

yields from the equation

$$
\begin{aligned}
& \left(\sin ^{-1} \theta \frac{\partial}{\partial \theta} \sin \theta \frac{\partial}{\partial \theta}+\sin ^{-2} \frac{\partial^{2}}{\partial \phi^{2}}\right) Y_{l m}(\zeta) \\
& =-l(l+1) Y_{l m}(\zeta) .
\end{aligned}
$$

Using the definition of spherical harmonics the Green function may be rewritten as

$$
\begin{aligned}
G= & -(4 \pi)^{-1} \sum_{l=1}^{\infty} \frac{1}{l(l+1)} \sum_{m=-l}^{m} \frac{(2 l+1)(l-m) !}{(l+m) !} \\
& \cdot P_{l}^{m}\left(u_{j}\right) P_{l}^{m}\left(u_{k}\right) e^{i m\left(\phi_{j}-\phi_{k}\right)} \\
\equiv & -(4 \pi)^{-1} \sum_{l=1}^{\infty} \frac{2 l+1}{l(l+1)} \sum_{m=-l}^{m} \frac{(l-m) !}{(l+m) !} \\
& \cdot P_{l}^{m}\left(u_{j}\right) P_{l}^{m}\left(u_{k}\right) \cos \left[m\left(\phi_{j}-\phi_{k}\right)\right] .
\end{aligned}
$$

Using the following result (see Landau and Lifshitz, p.697)

$P_{l}\left(\cos \beta_{j k}\right)=$

$=\sum_{m=-l}^{m} \frac{(l-m) !}{(l+m) !} P_{l}^{m}\left(u_{j}\right) P_{l}^{m}\left(u_{k}\right) \cos \left[m\left(\phi_{j}-\phi_{k}\right)\right]$,

we can obtain after some manipulations that

$$
\begin{aligned}
G\left(\cos \beta_{i j}\right) & =-\frac{1}{4 \pi} \sum_{l=1}^{\infty} \frac{2 l+1}{l(l+1)} P_{l}\left(\cos \beta_{i j}\right) \\
& =\frac{1}{4 \pi} \ln \left(1-\cos \beta_{i j}\right) .
\end{aligned}
$$

Here $\cos \beta_{j k}=u_{j} u_{k}+\left(1-u_{j}^{2}\right)^{1 / 2}\left(1-u_{k}^{2}\right)^{1 / 2} \cos \left(\phi_{j}-\right.$ $\left.\phi_{k}\right), \quad \beta_{j k}$ is the angle between two directions defined by the spherical angles $\theta_{j}, \phi_{j}$ and $\theta_{k}, \phi_{k}$.

The obtained formula follows from

$$
\begin{aligned}
\frac{d G(z)}{d z} & =-\frac{1}{4 \pi} \sum_{l=1}^{\infty} \frac{2 l+1}{l(l+1)} P_{l}^{\prime}(z) \\
& =-\frac{1}{4 \pi} \sum_{l=1}^{\infty}\left[\frac{1}{l} P_{l}^{\prime}(z)+\frac{1}{l+1} P_{l}^{\prime}(z)\right],
\end{aligned}
$$

where relations

$$
\begin{aligned}
& \left(1-z^{2}\right) P_{l}^{\prime}(z)=(l+1)\left[z P_{l}(z)-P_{l+1}(z)\right], \\
& \left(1-z^{2}\right) P_{l}^{\prime}(z)=-l z P_{l}(z)+l P_{l-1}(z)
\end{aligned}
$$

are used (here $P_{l}^{\prime}(z)=d P_{l}(z) / d z$ ). After the change of indices and the summation, the final expression (B6) is obtained. 


\section{Appendix C Canonical equations}

System (29) may be rewritten if it is necessary in the more convenient form using variables $\left(u_{s}=\cos \theta_{s}, \phi_{s}\right)$. In this case

$\gamma_{i} \partial_{t} u_{i}=-\partial_{\phi_{i}} \mathcal{H}_{i n t}, \quad \gamma_{i} \partial_{t} \phi_{i}=\partial_{u_{i}} \mathcal{H}_{\text {int }}$.

The canonical (Hamiltonian) equations of motion of $N$ point vortices on a sphere $\left(-1 \leq u_{i} \leq 1,-\infty \leq \phi_{i} \leq+\infty\right)$ can be formulated as

$$
\begin{aligned}
\gamma_{i} \partial_{t} u_{i}= & -\frac{\partial \mathcal{H}}{\partial \phi_{i}} \\
= & \gamma_{i} \sum_{k}^{\prime} \gamma_{k}\left(1-u_{i}^{2}\right)^{1 / 2}\left(1-u_{k}^{2}\right)^{1 / 2} . \\
& \cdot \mathcal{G}^{\prime}\left(\cos \beta_{i k}\right) \sin \left(\phi_{k}-\phi_{i}\right), \\
\gamma_{i} \partial_{t} \phi_{i}= & \frac{\partial \mathcal{H}}{\partial u_{i}} \\
= & \gamma_{i} \sum_{k}^{\prime} \gamma_{k}\left[u_{k}-\frac{u_{i}}{\left(1-u_{i}^{2}\right)^{1 / 2}}\left(1-u_{k}^{2}\right)^{1 / 2}\right] . \\
& \cdot \mathcal{G}^{\prime}\left(\cos \beta_{i k}\right) \cos \left(\phi_{k}-\phi_{i}\right) .
\end{aligned}
$$

Here,

$\cos \beta_{j k}=u_{j} u_{k}+\left(1-u_{j}^{2}\right)^{1 / 2}\left(1-u_{k}^{2}\right)^{1 / 2} \cos \left(\phi_{j}-\phi_{k}\right)$,

and $\beta_{j k}$ is the angle between two directions which define the locations of $j$ - and $k$-vortices on the sphere.

\section{References}

Abramowitz, M. and Stegun, I. A., Handbook of Mathematical Functions, U. S. Govt. Printing Office, Washington D. C., 1964.

Aref, H., Integrable, chaotic, and turbulent vortex motion in twodimensional flows, Ann. Rev. Fluid Mech., 15, 345-389, 1983.

Bogomolov, V. A., The dynamics of vorticity on a sphere, Izv. Akad. Nauk SSSR, Mekh. Ghid. Gaza, 13, 57, 1977.

Goncharov, V. P. and Pavlov, V. I., Problems of Hydrodynamics in
Hamiltonian Approach, Moscow Univ., Moscow, 1993 (in Russian).

Goncharov, V. P. and Pavlov, V. I., Some remarks on the physical foundation of the Hamiltonian description of fluid motions, Eur J. Mech., B/Fluids, 16, no. 4, 509-555, 1997.

Goncharov, V. P. and Pavlov, V. I., Vortex structures generated by a coastal current in harbour-like basins at large Reynolds number, J. Fluid Mech., 351, 201-221, 1997.

Goncharov, V. and Pavlov, V., On the Hamiltonian approach: Applications to geophysical flows, Nonlinear Processes in Geophysics, 5, 219-240, 1998.

Holton, J. R., An Introduction to Dynamic Meteorology, 3th ed., Academic Press, 1992.

Lamb, H., Hydrodynamics, 6th ed., Cambridge University Press, Cambridge, 1932, (reprinted by Dover, New York, 1945).

Landau, L. D. and Lifshitz, E. M., Physique theorique: Mecanique Quantique, IYeme ed., Mir, Moscou, 1982.

Landau, L. D. and Lifshitz, E. M., Fluid Mechanics, 2nd ed., rev., Pergamon Press, Oxford, 1987.

McIntyre, M. I., Atmospheric dynamics: some fundamentals with observational implications, in Proc. Int. School of Physics "Enrico Fermi”, CXV Course, (ed. J. C. Gille and G. Visconti), 1991.

Marcus, P. S., Vortex dynamics in a shearing zonal flow, J. Fluid Mech., 215, 393-430, 1990.

Morse, P.M. and Feshbach, H., Methods of Theoretical Physics, McGraw-Hill, 1953.

Novikov, E. A., Generalized dynamics of three-dimensional vorticial singularities (vortons), Sov. Phys. - JETP, 57, (3), 566-569, 1983.

Obukhov, A. M., On the question of geostrophic wind, Izv. Akad. Nauk SSSR, Ser. Geofiz., 8, no. 4, 281-306, 1949.

Overman, E. A. and Zabusky, N. J., Evolution and merger of isolated vortex structures, Phys. Fluids, 25, 1297-1305, 1982.

Pedlosky, J., Geophysical Fluid Dynamics, Springer-Verlag, New York, 1987.

Polvani, L. M. and Dritschel, D. G., Wave and vortex dynamics on the surface of a sphere, J. Fluid Mech., 255, 35-64, 1993.

Reznik, G. M., Dynamics of singular vortices on a beta-plane, J. Fluid Mech., 240, 405-432, 1992.

Zabusky, N. J. and McWilliams, J. S., A modulated point vortex for geostrophic beta-plane dynamics, Phys. Fluids, 25, 2175, 1982. 